\title{
Are Hounsfield Unit Measurements of Bony Absorption Changes in Cholesteatoma Helpful?
}

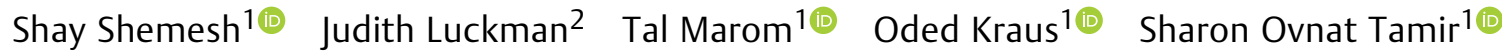 \\ ${ }^{1}$ Department of Otolaryngology-Head and Neck Surgery, Samson \\ Assuta Ashdod University Hospital, Faculty of Health Sciences, Ben \\ Gurion University, Ashdod, Israel \\ 2 Department of Radiology, Assuta Medical Centers, Faculty of Health \\ Sciences, Ben Gurion University, Tel Aviv, Israel \\ Address for correspondence Sharon Ovnat Tamir, MD, Department of \\ Otolaryngology-Head and Neck Surgery, Samson Assuta Ashdod \\ University Hospital, Faculty of Health Sciences, Ben Gurion University, \\ 7 Ha’Refua Street, 7747629, Ashdod, Israel \\ (e-mail: sharono@assuta.co.il).
}

Int Arch Otorhinolaryngol 2022;26(3):e414-e421.

\begin{abstract}
Introduction High-resolution computed tomography (HRCT) scans of the temporal bone are used to assess the bony erosion of the middle-ear structures whenever cholesteatoma is suspected.

Objective To study the differences in HRCT Hounsfield unit (HU) index measurements of middle-ear bony structures between an ears with and without cholesteatoma.

Methods A retrospective study of 59 patients who underwent surgery due to unilateral cholesteatoma. The HRCT HU index of the scutum, of three middle-ear ossicles, of the lateral semicircular canal (LSCC), and of the fallopian canal was measured in both ears. A comparison was made between the cholesteatoma and the non-cholesteatomatous ear (control). All measurements were conducted by an otolaryngologist. To assess the interobserver bias, $10 \%$ of the samples were randomly and independently assessed by another otolaryngologist and a neuroradiologist who were blinded.

Results The average HU index was lower in the ear with cholesteatoma when compared with the non-cholesteatomatous ear. While the differences were statistically significant regarding the measurements of the scutum $(516.02 \pm 311.693$ versus $855.64 \pm 389.999$; $p=0.001)$, the malleus $(1049.44 \pm 481.765$ versus $1413.47 \pm 313.376 ; p=0.01)$, and the incus $(498.03 \pm 264.184$ versus $714.25 \pm 405.631 ; p=0.001)$, the differences in the

\section{Keywords}

- computed tomography

- Hounsfield unit

- cholesteatoma

- ossicles measurements of the $\operatorname{LSCC}(1042.34 \pm 301.066$ versus $1154.53 \pm 359.609 ; p=0.69)$ and of the fallopian canal $(467.19 \pm 221.556$ versus $543.51 \pm 263.573 ; p=0.108)$ were not significantly different between both groups. The stapes was immeasurable in both groups due to its small size.

Conclusion Hounsfield unit index measurements are a useful tool that may aid in the diagnosis of early-stage cholesteatoma.
\end{abstract}

received

July 3, 2020

accepted after revision

March 5, 2021

published online

November 11, 2021
DOI https://doi.org/ $10.1055 / \mathrm{s}-0041-1736580$ ISSN 1809-9777.

\footnotetext{
(c) 2021. Fundação Otorrinolaringologia. All rights reserved. This is an open access article published by Thieme under the terms of the Creative Commons Attribution-NonDerivative-NonCommercial-License, permitting copying and reproduction so long as the original work is given appropriate credit. Contents may not be used for commercial purposes, or adapted, remixed, transformed or built upon. (https://creativecommons.org/ licenses/by-nc-nd/4.0/) Thieme Revinter Publicações Ltda., Rua do Matoso 170, Rio de Janeiro, RJ, CEP 20270-135, Brazil
} 


\section{Introduction}

Cholesteatoma is a disease characterized by a slowly growing epidermal cyst that is entrapped within the temporal bone and secretes proteolytic and osteolytic enzymes. ${ }^{1}$ Most frequently, it affects the middle-ear ossicles (specially the incus), ${ }^{2}$ the tegmen tympani and tegmen mastoideum, the semicircular canal(s) (most frequently the lateral semicircular canal, LSCC), and the tympanic segment of the fallopian canal. $^{3}$ The diagnosis is based on otoscopic findings, which typically demonstrate a keratinizing epithelial invasion into the middle-ear cleft. ${ }^{4}$ Prior to surgery, when devising the surgical plan, preoperative diagnostic imaging studies are performed to confirm the presence of cholesteatoma and determine its extension. Such studies include a high-resolution computed tomography (HRCT) scan and a magnetic resonance imaging (MRI) scan of the temporal bone. ${ }^{5}$

In most cases, HRCT is the imaging modality of choice. ${ }^{6}$ Another imaging modality that may be employed is the MRI, which is better for characterizing soft tissue and detecting intracranial complications. ${ }^{7}$ The typical findings of cholesteatoma on an HRCT scan include middle-ear and/or mastoid fullness with variable adjacent bony erosion of the scutum, the middle-ear ossicles, the semicircular canal(s), the fallopian canal, and the tegmen tympani/mastoideum. ${ }^{8}$ Tissue radiodensity is measured with the Hounsfield unit (HU) scale. The calculated value is a linear transformation of the original measurement of the linear attenuation coefficient, in which the radiodensity of distilled water at standard pressure and temperature is defined as zero. ${ }^{9}$ Each biological tissue has its own unique HU property. For example, water $=0$, air $=-1,000$, fat tissue $=-120$ to -90 , muscle tissue $=+35$ to +55 , and the skull (bone) $>+200$.

To date, there is no routine practice regarding the use of $\mathrm{HU}$ measurements in otology. There are few studies on the use of $\mathrm{HU}$ to differentiate cholesteatoma from other chronic inflammatory tissues, but their results are conflicting. We presume that the bony HU density index can be used as a simple and feasible tool in the assessment of bony demineralization caused by cholesteatoma. This can be very helpful in determining the stage of the disease, its involvement, and influence the decision regarding the surgery. In the present study, we measured several bony sites commonly affected by cholesteatoma in ears with the disease and compared them to the contralateral non-cholesteatomatous ears to obtain information regarding the changes in bony density caused by cholesteatoma.

\section{Materials and Methods}

\section{Objective}

To examine if there are statistically significant differences in the average $\mathrm{HU}$ measurements of the scutum, the three middle-ear ossicles, the LSCC, and the fallopian canal of the facial nerve in preoperative HRCT studies of patients presenting with unilateral cholesteatoma of the middle-ear cleft (study group), in comparison to the same patients' contralateral non-cholesteatomatous ("healthy") ear (control group).

\section{Study Design}

The present was a retrospective chart review which included adult patients ( $>18$ years of age) who presented with unilateral middle-ear cleft cholesteatoma (International Classification of Diseases-9 [ICD-9] codes: 385.3 cholesteatoma of middle ear and mastoid; 385.30 cholesteatoma unspecified; 385.31 cholesteatoma of attic; 385.32 cholesteatoma of middle ear; and 385.33 cholesteatoma of middle ear and mastoid) and underwent any form of mastoidectomy (ICD-9 codes: 20.49 other mastoidectomy; 20.41 simple mastoidectomy; 20.42 radical mastoidectomy; and 20.4 mastoidectomy).

\section{Patients}

The patients' demographics, side of the affected ear, and HRCTs were retrieved from the electronic medical records.

\section{Inclusion Criteria}

- Adult patients with unilateral cholesteatoma who underwent mastoidectomy, with the surgeon's intraoperative description of the cholesteatoma and/or confirmation of the cholesteatoma in the final pathological report.

- Patients with anatomically normal contralateral noncholesteatomatous ("healthy") ear.

- Patients with preoperative HRCT images available for inspection in our radiological archives.

\section{Exclusion Criteria}

- Patients who had previously undergone otological surgery (except for ventilating tube insertion).

- Patients with congenital inner-ear anomalies (that is, Mondini dysplasia, Michel aplasia, or enlarged vestibular aqueduct).

- Patients with bilateral cholesteatoma.

- Patients lacking preoperative HRCT scans in our radiological archives.

We initially retrieved 623 medical files of patients matching the above ICD-9 codes between 2008 and 2018. Of them, 523 patients were excluded because they did not meet our inclusion criteria. The remaining 100 eligible patients, diagnosed with unilateral suspected cholesteatoma prior to surgery, were subjected to a further review. After reviewing the HRCT scans of these patients, 41 patients were excluded because the thickness of their HRCT slide failed to meet the requirements (slide thickness $>0.7 \mathrm{~mm}$ ); thus, 59 patients

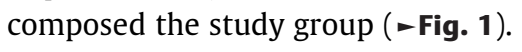

\section{Measurements and Calculations}

We performed repeated measurements of the HU index in ears with cholesteatoma and compared them to those of the contralateral non-cholesteatomatous ("healthy") ear of the same patient. All measurements were performed on a noncontrast enhanced coronal HRCT scan of the temporal bone (using two different scanners: one from GE Medical Systems, Milwaukee, WI. US, and the other from Siemens, Munich, Germany) with a slide thickness ranging from $0.5 \mathrm{~mm}$ to $0.7 \mathrm{~mm}$. The HU index was measured in a coronal-plane 


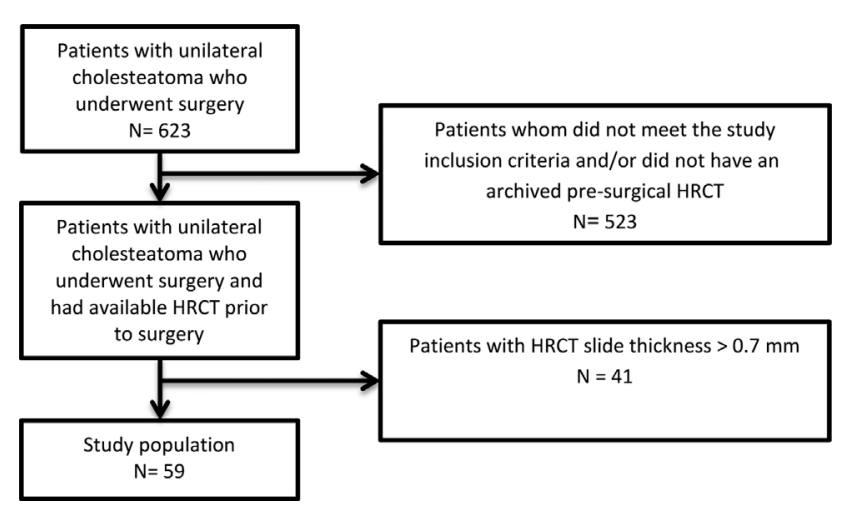

Fig. 1 Flowchart of the study population.

HRCT scan of the temporal bone with the same slide thickness after reconstruction.

For each ear, we performed 6 different measurements, at the level of: 1) the scutum; 2) the malleolar head; 3 ) the long process of the incus; 4) the stapes; 5) the LSCC; and 6) the fallopian canal. These areas were chosen because they are known to be prone to cholesteatoma invasion and erosion. ${ }^{6}$

To obtain the most reliable measurements, we did not only sample these regions of interest (ROIs), but also added two additional measurements which were obtained one slide above and one below the ROI. All ROIs were identical, with a surface area of $0.1 \mathrm{~mm}^{2}$. Following the three sequential measurements of the ROI, we calculated their average, and compared the values with three identical average ROI values in the contralateral non-cholesteatomatous ear.

All measurements were conducted by one otolaryngologist (S.S.), to confirm the validity of the HU index measurements (interobserver bias); $10 \%$ of the samples were randomly and independently assessed by a senior neuroradiologist (J.L.) and a senior otolaryngologist (S.O.T.). A Cronbach alpha test was used to evaluate the interobserver bias rate to assess the reliability of the HU index measurements.

\section{Statistical Analysis}

The data were recorded on a Microsoft Excel (Microsoft Corp., Redmond, WA, US) spreadsheet. The unit of analysis was the temporal-bone HRCT of patients with unilateral cholesteatoma. The affected ear was compared with the "healthy" ear (control). The statistical analysis was performed using the Statistical Package for the Social Sciences (IBM SPSS Statistics for Windows, IBM Corp., Armonk, NY, US) software, version 23.0. The reliability of the HU index to test the interobserver bias was assessed with an Cronbach alpha test, and the Mann-Whitney $U$ test was used for the null hypothesis. The results of every test were considered statistically significant $(p<0.05)$.

\section{Results}

\section{Demographics}

In total, 59 patients were enrolled in the study. Of them, 34 (58\%) were male, and 25 (42\%) were female, with ages ranging from 18 to 69 years (mean \pm standard deviation
[SD]: $32.5 \pm 15.33)$. Regarding laterality, 27 (46\%) patients had cholesteatoma on the right side, while $32(54 \%)$, on the left side.

\section{Measurements}

In the cholesteatoma group, the mean $\mathrm{HU}$ value for the scutum was of $516.02 \pm 311.693$ (range: 134 to 1,557; $p=0.001)$; for the malleolar head, it was of $1,049.44 \pm 481.765$ (range: 198 to 2,$232 ; p=0.01$ ); and, for the long process of the incus, it was of $498.03 \pm 264.184$ (range: 208 to $1391 ; p=0.001$ ).

In the non-cholesteatoma (control) group, the mean $\mathrm{HU}$ value for the scutum was of $855.64 \pm 389.999$ (range: 202 to 1,$881 ; p=0.001$ ); for the malleolar head, it was of $1,413.47 \pm 313.376$ (range: 330 to 1,$975 ; p=0.01$ ); and, for the long process of the incus, it was of $714.25 \pm 405.631$ (range: 206 to $1910 ; p=0.001$ ). The differences in the measurements of the stapes, the LSCC and the fallopian canal were not statistically significant.

\section{Interobserver Differences}

The results of the interobserver reliability test (calculated using a Cronbach alpha test) were acceptable for the scutum $(0.831)$ and the malleus $(0.860)$ in both groups. All of the other test for the comparison of ROI measurements had results $<0.7$ in the Cronbach alpha test, which implies that there were inconsistencies between investigators. Regardless, when comparing the cholesteatomatous and non-cholesteatomatous sides, our results indicated a statistically significant difference for the scutum, the malleus and the incus $(p=0.01)$, with lower values on the HU index for all regions in the study group when compared with the control group. As for the stapedial measurements of several "healthy" ears, we obtained negative $\mathrm{HU}$ values, indicating the measurements were performed on air instead of bone. Additionally, in the study group, the $\mathrm{HU}$ index values for the stapes were $\sim+90$, again indicating that the actual measurement was of the cholesteatoma itself or of the air surrounding the stapes, instead of the stapes itself.

- Tables 1,2,3 show a comparison of $\mathrm{HU}$ index values between the two groups ( - Table $\mathbf{1}$ ), as well as a comparison according to the thickness of the HRCT slide ( - Table 2 ) and gender ( - Table 3). - Fig. 2A-F shows the differences in HU index values between the ears with and without cholesteatoma in individual bony structures of the middle-ear cleft. - Fig. 3A-B shows the HU distribution range and the differences between the two groups.

\section{Discussion}

In the present study, we examined if there were statistically significant differences in HU measurements on HRCT scans in six common sites of involvement in patients with unilateral cholesteatoma, by comparing the affected ear with the contralateral "healthy" ear. In our analysis of 59 patients with HRCT scans with a slide thickness $\leq 0.7 \mathrm{~mm}$, we were able to demonstrate significantly lower HU index measurements in the scutum, malleus and incus of affected ears when 
Table 1 Comparison of Hounsfield Unit Index measurements

\begin{tabular}{|l|l|l|l|l|l|l|l|}
\hline Ear & Sites Inspected & N & Minimum & Maximum & Mean & $\begin{array}{l}\text { Standard } \\
\text { deviation }\end{array}$ & -value \\
\hline Cholesteatomatous & Scutum & 59 & 134 & 1,557 & 516.01 & 311.69 & 0.01 \\
\cline { 2 - 8 } & Malleus & 59 & 198 & 2,232 & $1,049.44$ & 481.77 & 0.01 \\
\cline { 2 - 7 } & Incus & 59 & 208 & 1,391 & 498.03 & 264.18 & 0.01 \\
\cline { 2 - 8 } & Stapes & 59 & -14 & 512 & 124.76 & 108.72 & Not available \\
\cline { 2 - 7 } & Lateral semicircular canal & 59 & 217 & 1,652 & $1,042.33$ & 301.07 & 0.69 \\
\cline { 2 - 7 } & Fallopian canal & 59 & 215 & 1,282 & 467.18 & 221.56 & 0.108 \\
\hline \multirow{5}{*}{ Non-cholesteatomatous } & Scutum & 59 & 202 & 1,881 & 855.64 & 390.00 & 0.01 \\
\cline { 2 - 8 } & Malleus & 59 & 330 & 1,975 & $1,413.47$ & 313.38 & 0.01 \\
\cline { 2 - 7 } & Incus & 59 & 206 & 1,910 & 714.25 & 405.63 & 0.01 \\
\cline { 2 - 7 } & Stapes & 59 & -855 & -21 & -350.40 & 193.23 & Not available \\
\cline { 2 - 7 } & Lateral semicircular canal & 59 & 140 & 2,013 & $1,154.52$ & 359.61 & 0.69 \\
\cline { 2 - 6 } & Fallopian canal & 59 & 208 & 1,283 & 543.50 & 263.57 & 0.108 \\
\hline
\end{tabular}

Note: The minimum, maximum and mean values are expressed in Hounsfield units.

Table 2 Comparison of Hounsfield Unit measurements obtained by high-resolution computedrized tomography according to slide thickness

\begin{tabular}{|c|c|c|c|c|c|c|}
\hline \multicolumn{2}{|c|}{ Slide thickness } & \multirow{2}{*}{$\frac{N}{8}$} & \multirow{2}{*}{$\begin{array}{l}\text { Minimum } \\
210\end{array}$} & \multirow{2}{*}{$\begin{array}{l}\text { Maximum } \\
1,820\end{array}$} & \multirow{2}{*}{$\begin{array}{l}\text { Mean } \\
1,120.13\end{array}$} & \multirow{2}{*}{$\begin{array}{l}\text { Standard deviation } \\
572.554\end{array}$} \\
\hline $0.5 \mathrm{~mm}$ & Scutum & & & & & \\
\hline & Malleus & 8 & 354 & 1,712 & 1236.88 & 402.498 \\
\hline & Incus & 8 & 212 & 1,355 & 613.88 & 359.099 \\
\hline & Stapes & 8 & -521 & 245 & -136.88 & 301.739 \\
\hline & Lateral semicircular canal & 8 & 746 & 1,422 & $1,120.75$ & 251.145 \\
\hline & Fallopian canal & 8 & 227 & 1,283 & 579.25 & 397.073 \\
\hline \multirow[t]{6}{*}{$0.6 \mathrm{~mm}$} & Scutum & 72 & 206 & 1,850 & 640.47 & 341.535 \\
\hline & Malleus & 72 & 222 & 2,232 & $1,252.54$ & 487.655 \\
\hline & Incus & 72 & 206 & 1,712 & 560.19 & 303.824 \\
\hline & Stapes & 72 & -744 & 457 & -115.49 & 295.524 \\
\hline & Lateral semicircular canal & 72 & 140 & 1,773 & 1080.42 & 329.080 \\
\hline & Fallopian canal & 72 & 223 & 1,282 & 537.60 & 244.417 \\
\hline \multirow[t]{6}{*}{$0.7 \mathrm{~mm}$} & Scutum & 38 & 134 & 1,881 & 680.34 & 389.892 \\
\hline & Malleus & 38 & 198 & 1,874 & $1,190.37$ & 365.845 \\
\hline & Incus & 38 & 222 & 1,910 & 691.58 & 437.556 \\
\hline & Stapes & 38 & -855 & 512 & -102.71 & 268.368 \\
\hline & Lateral semicircular canal & 38 & 233 & 2,013 & $1,127.87$ & 365.331 \\
\hline & Fallopian canal & 38 & 208 & 959 & 428.68 & 191.797 \\
\hline
\end{tabular}

Note: The minimum, maximum and mean values are expressed in Hounsfield units.

compared with "healthy ears". We had authors from two different disciplines (two otolaryngologists and one neuroradiologist) measuring the $\mathrm{HU}$ index values who were blinded to the results, to decrease the bias. The Cronbach alpha test for interobserver reliability revealed acceptable values for the scutum and the malleus in both groups, whereas all of the other regions measured yielded low results, which implies that there were inconsistencies between the investigators regarding the ROI measurements. Nevertheless, when we compared both ears, our results indicated a statistically significant difference for the scutum, the malleus and the incus, and lower HU index values for all regions in the study group when compared with the control group. The stapedial HU index measurements were 
Table 3 Comparison of Hounsfield Unit measurements obtained by high-resolution computedrized tomography according to gender

\begin{tabular}{|c|c|c|c|c|c|c|c|c|}
\hline & \multirow[b]{2}{*}{ Gender } & \multirow[b]{2}{*}{$\mathbf{N}$} & \multicolumn{3}{|c|}{ Study group } & \multicolumn{3}{|c|}{ Control group } \\
\hline & & & Mean & $\begin{array}{l}\text { Standard } \\
\text { deviation }\end{array}$ & $p$-value & Mean & $\begin{array}{l}\text { Standard } \\
\text { deviation }\end{array}$ & $p$-value \\
\hline \multirow[t]{2}{*}{ Scutum } & Male & 34 & 466.82 & 253.67 & & 815.29 & 347.96 & \\
\hline & Female & 25 & 582.92 & 371.78 & 0.185 & 918.15 & 451.75 & 0.334 \\
\hline \multirow[t]{2}{*}{ Malleus } & Male & 34 & 975.26 & 518.94 & & $1,357.06$ & 342.44 & \\
\hline & Female & 25 & $1,150.32$ & 415.00 & 0.156 & $1,468.52$ & 258.64 & 0.153 \\
\hline \multirow[t]{2}{*}{ Incus } & Male & 34 & 485.91 & 303.15 & & 709.88 & 378.32 & \\
\hline & Female & 25 & 514.52 & 204.48 & 0.667 & 720.96 & 431.69 & 0.917 \\
\hline \multirow[t]{2}{*}{ Stapes } & Male & 34 & 108.76 & 95.95 & & -354.53 & 200.82 & \\
\hline & Female & 25 & 146.52 & 122.67 & 0.208 & -308.81 & 222.92 & 0.410 \\
\hline \multirow[t]{2}{*}{ Lateral semicircular canal } & Male & 34 & $1,060.65$ & 332.03 & & $1,155.26$ & 386.68 & \\
\hline & Female & 25 & $1,017.44$ & 257.56 & 0.576 & $1,158.48$ & 319.61 & 0.972 \\
\hline \multirow[t]{2}{*}{ Fellopian canal } & Male & 34 & 417.91 & 184.23 & & 500.68 & 218.25 & \\
\hline & Female & 25 & 534.20 & 252.67 & 0.058 & 594.00 & 299.90 & 0.182 \\
\hline
\end{tabular}

Note: The minimum, maximum and mean values are expressed in Hounsfield units.
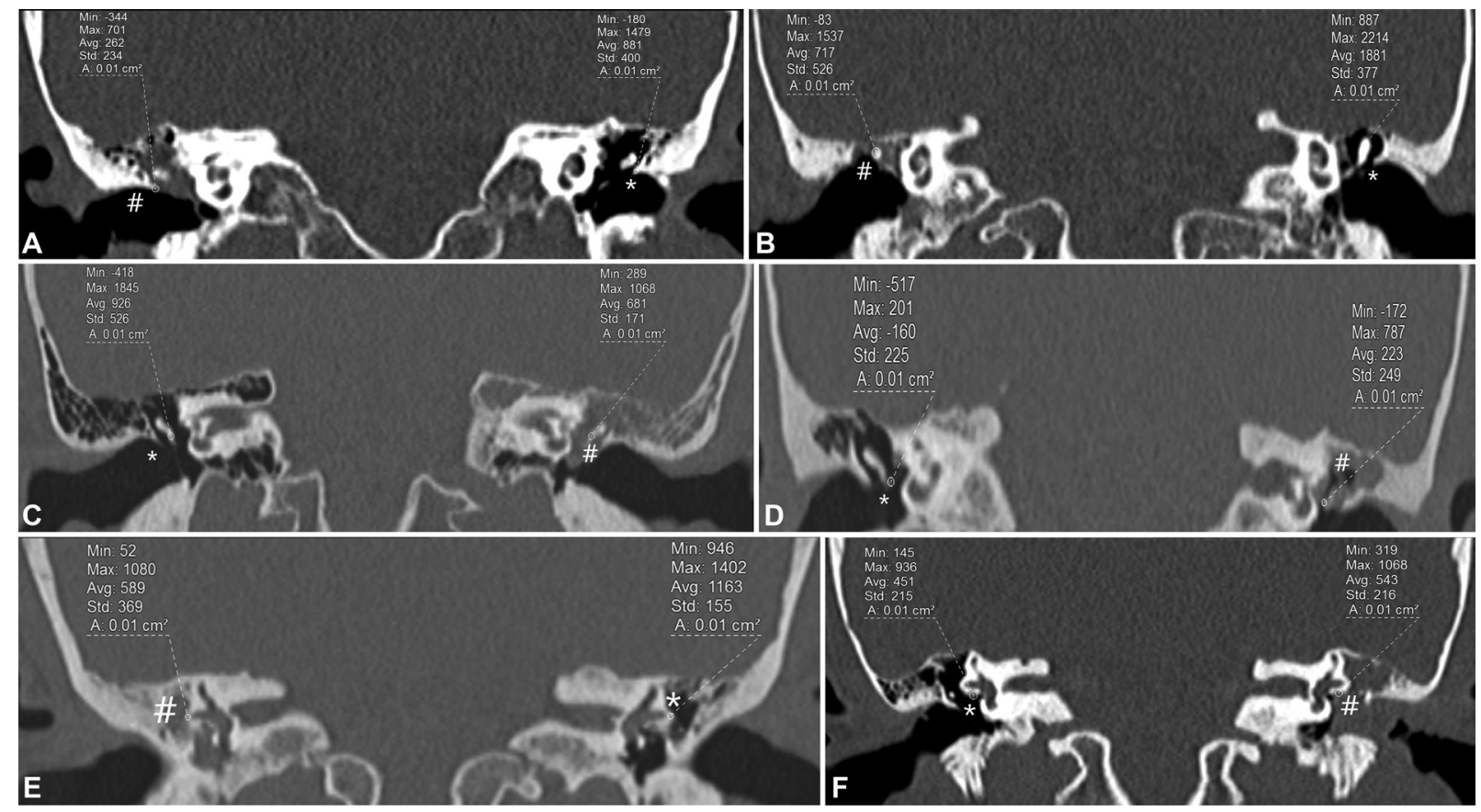

Fig. 2 (A-F): Coronal HRCT scans of the temporal bone without contrast material, demonstrating the differences in $\mathrm{HU}$ index values between ears with and without cholesteatoma. (A) Right scutum in a cholesteatomatous ear: $\mathrm{HU}=262$; left unaffected scutum: $\mathrm{HU}=881$. (B) Right malleolar head in a cholesteatomatous ear: $\mathrm{HU}=717$; left unaffected malleolar head: $\mathrm{HU}=1,881$. (C) Left long process of the incus in a cholesteatomatous ear: $\mathrm{HU}=681$; right unaffected long process of the incus: $\mathrm{HU}=926$. (D) Left stapes in a cholesteatomatous ear: $\mathrm{HU}=223$; right unaffected stapes: $\mathrm{HU}=-160$. (E) Right $L S C C$ in a cholesteatomatous ear: $\mathrm{HU}=589$; left unaffected LSCC: $\mathrm{HU}=1,163$. (F). Left fallopian canal in a cholesteatomatous ear: $\mathrm{HU}=543$; right unaffected fallopian canal: $\mathrm{HU}=451$.

unreliable; they were inconsistent, and were performed on the surrounding region instead of on the stapes itself. This result is not surprising, considering that the size of the stapes superstructure is very small, around $1 \mathrm{~mm},{ }^{10}$ which makes the HU index sampling difficult, consequently making the stapes measurements redundant for the clinical judgement.
As for the LSCC and the fallopian canal, our results indicated that there were no statistically significant differences between the two groups. The reason is probably because, in most sampled cases, there was no involvement/invasion of the LSCC, and for the fallopian canal, it is known that, in 55\% of "healthy" ears, the canal is partially exposed because of 

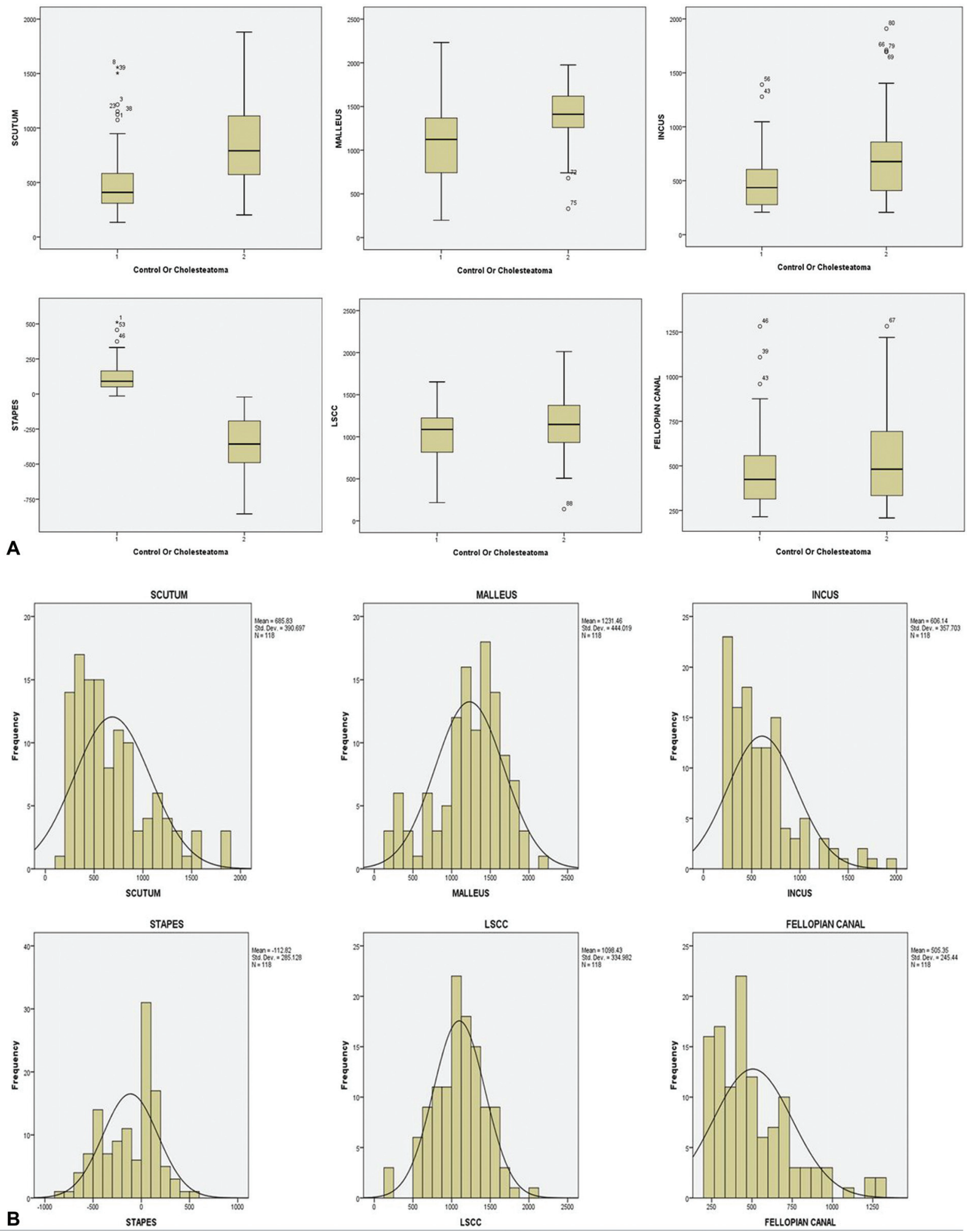

Fig. 3 (A) HU index distribution differences between the study and control groups. (B) HU index distribution range of the study and control groups.

bony dehiscence, ${ }^{4}$ denoting possible changes in HU index values in the "healthy" ear as well.

Previous studies that investigated the use of $\mathrm{HU}$ values to differentiate cholesteatoma from other middle-ear pathologies demonstrated only scant data on the subject, but none investigated the HU differences in bony radiodensity in the middle-ear cleft of patients presenting with cholesteatoma.

In a retrospective study from South Korea published in 2011, Park et al. ${ }^{11}$ investigated the differences in HU index values between cholesteatoma and chronic otitis media 
(COM) at the aditus ad antrum. Their sample was composed of 82 patients divided into 2 groups: group 1 had pathologically-confirmed cholesteatoma, and group 2 presented with middle-ear granulation tissue. The HU index was measured at the antrum in an axial plane, at the level of the LSCC. Each HU measurement was performed in three different locations: at the level of the suspected focal points, and one slide above and one below the first measurement respectively. The ROI measured $2.49 \mathrm{~mm}^{2}$, and all measurements were independently performed by 2 investigators unexposed to the patient's final diagnosis. The lowest of the three measurements was chosen. The HU was calculated as $42.68 \pm 24.42$ in the cholesteatomatous group and as $86.07 \pm 26.50$ in the non-cholesteatomatous group. The differences between the groups were statistically significant. The authors concluded that, by measuring the HU index, the sensitivity and specificity to diagnose cholesteatoma could be improved.

On the other hand, in a retrospective study published in 2012, Lee et al. ${ }^{12}$ measured the differences in the HU index in preoperative HRCT scans of COM and cholesteatoma patients. The sample was composed of 91 patients who underwent tympanomastoidectomy. The pathological findings were described by the surgeon, and later on, classified by histopathology as cholesteatoma, granulation tissue, cholesterol granuloma or effusion. The HU index of all pathological findings was independently measured by three different otolaryngologists. The results showed that the mean HU values for the cholesteatomatous lesions were between 35.7 and $66.6 \mathrm{HU}$, and those for the non-cholesteatomatous lesions were between 32.9 and 51.3 HU. No statistically significant difference was found between the two types of lesion $(p=0.305)$. Furthermore, the Cronbach alpha test demonstrated that there was a significant difference in the HU measurement levels among the three examiners, and the authors concluded that the HU index was impractical for clinical purposes.

In another study from South Korea published in 2014, Ahn et al. $^{13}$ investigated the differences in the HU index on preoperative coronal HRCT slides of the temporal bone in patients with congenital cholesteatoma and COM. The study included 43 children who underwent surgery: 21 for congenital cholesteatoma, confirmed by postoperative histopathological findings, and 22 for COM. The study showed a mean HU of $37.36 \pm 6.11$ in the congenital cholesteatoma group, and of $76.09 \pm 8.74$ in the COM group $(p<0.001)$. The cut-off value between the two groups was of 55.5; thus, the authors concluded that $\mathrm{HU}$ index values $<55.5$ represented congenital cholesteatoma, and higher values were consistent with COM. They also concluded that the HU measurement may be useful as an additional indicator to diagnose congenital cholesteatoma.

Concerning the distribution range of the HU index, the study demonstrated a wide range of values for all measured ROIs. One reason, as shown in a study by Groell et al., ${ }^{14}$ might be the variations in the HU index values according to different CT scanners, in the reconstruction algorithms, in slice thickness, and in dosage. Furthermore, in a study by Davis et al., ${ }^{15}$ the HRCT reconstruction algorithm had a significant effect on HU index values obtained from the same CT scanner. Another reason could have been the HU sampling method. The HU values can be measured either by cursor or by encircling the ROI. The advantage of the cursor is its accuracy, but the disadvantage is its inability to sample more than one dot per image, which makes sampling with this method inaccurate due to bias. For that reason, we decided to use the ROI encircling method. To increase the accuracy, we used the same value for the ROI surface area in every measurement. Despite our efforts, our results showed a fairly wide HU index distribution range, which is similar to the study by Lee et al., ${ }^{12}$ who measured a ROI of $1.5 \mathrm{~mm}^{2}$ and still demonstrated a wide variation.

To our knowledge, the present is the first study on the measurement of HU index values of the bony structures in the middle-ear cleft to compare the ear affected with cholesteatoma to the unaffected ear of the same patient.

Additionally, the strengths of our study are: 1) the values of the interobserver reliability were acceptable for the scutum and malleus in both groups, and the results for the scutum, malleus and incus were statistically significant; 2) the large sample of patients with unilateral cholesteatoma who underwent HRCT; 3 ) the measurement of the HU index of the most frequently-involved middleear bony structures; 4) the simplicity of the measurement, which is available in every HRCT viewer program, without the need for a special protocol; 5) the fact that the authors who measured the HU index values were from different disciplines (two otolaryngologists and one neuroradiologist), and were blinded to the results, thus decreasing the chance for bias; and 6) the comparison of the values in the same patient might decrease the chances of bias arising from HU index measurments performed in different individuals.

We acknowledge several limitations of our work: 1) the data was collected from several centers with different HRCT machines/protocols, potentially leading to variations in the HU index measurements; and 2) the variation in the thickness of the HRCT slides were included in the study, leading to a variation in $\mathrm{HU}$ index measurements.

\section{Conclusion}

The present study indicates that the HU index may be a useful tool to support the early diagnosis of unilateral cholesteatoma, especially in cases when erosion of the scutum, malleus or incus is suspected.

\section{Data Availability}

The data that support the findings of the present study can be made available by the corresponding author upon reasonable request.

\section{Compliance with Ethical Standards}

1. Funding: the present research did not receive any specific grant from funding agencies in the public, private or not-for-profit sectors. 
2. Ethical approval: the present study was approved by the Samson Assuta Ashdod University Hospital Institutional Review Board (under protocol number: 00034-18-AAA) and the Assuta Medical Centers Institutional Review Board (under protocol number: 0091-18-ASMC).

\section{Conflict of Interests}

Tal Marom is a medical advisor of Novus Therapeutics, Inc., Irvine, CA, US. The other authors have no conflict of interests to declare.

\section{References}

1 Kuo CL, Shiao AS, Yung M, et al. Updates and knowledge gaps in cholesteatoma research. BioMed Res Int 2015;2015:854024

2 Akarcay M, Kalcioglu MT, Tuysuz O, Timurlenk E, Guclu H. Ossicular chain erosion in chronic otitis media patients with cholesteatoma or granulation tissue or without those: analysis of 915 cases. Eur Arch Otorhinolaryngol 2019;276(05):1301-1305

3 Kalcioglu MT, Kilic O, Tuysuz O, Serifler S, Tekin M. Facial canal dehiscence rate: a retrospective analysis of 372 chronic otitis media cases. Eur Arch Otorhinolaryngol 2019;276(01):79-83

4 Paul W. Flint M. Cummings Otolaryngology Head and Neck Surgery. 6th ed 2015

5 Ilıca AT, Hıdır Y, Bulakbaşı N, et al. HASTE diffusion-weighted MRI for the reliable detection of cholesteatoma. Diagn Interv Radiol 2012;18(02):153-158

6 Baráth K, Huber AM, Stämpfli P, Varga Z, Kollias S. Neuroradiology of cholesteatomas. AJNR Am J Neuroradiol 2011;32(02):221-229

7 Vercruysse JP, De Foer B, Pouillon M, Somers T, Casselman J, Offeciers $E$. The value of diffusion-weighted MR imaging in the diagnosis of primary acquired and residual cholesteatoma: a surgical verified study of 100 patients. Eur Radiol 2006;16(07): 1461-1467

8 Walshe P, McConn Walsh R, Brennan P, Walsh M. The role of computerized tomography in the preoperative assessment of chronic suppurative otitis media. Clin Otolaryngol Allied Sci 2002;27(02):95-97

9 Hounsfield GN. Computed medical imaging. Nobel lecture, Decemberr 8, 1979. J Comput Assist Tomogr 1980;4(05): 665-674

10 aWengen DF, Nishihara S, Kurokawa H, Goode RL. Measurements of the stapes superstructure. Ann Otol Rhinol Laryngol 1995;104 (4 Pt 1):311-316

11 Park MH, Rah YC, Kim YH, Kim JH. Usefulness of computed tomography Hounsfield unit density in preoperative detection of cholesteatoma in mastoid ad antrum. Am J Otolaryngol 2011; 32(03):194-197

12 Lee DH, Kim CS, Park CW, Chung DY. Is preoperative computed tomographic density measurement of soft tissues helpful in the diagnosis of cholesteatoma? Ann Otol Rhinol Laryngol 2012;121 (12):792-797

13 Ahn SH. Usefulness of Computed Tomography Hounsfield Unit Measurement for Diagnosis of Congenital Cholesteatoma. In: Kim Y-W, editor:: J Korean Soc Radio; 2014

14 Groell R, Rienmueller R, Schaffler GJ, Portugaller HR, Graif E, Willfurth P. CT number variations due to different image acquisition and reconstruction parameters: a thorax phantom study. Comput Med Imaging Graph 2000;24(02):53-58

15 Davis AT, Palmer AL, Pani S, Nisbet A. Assessment of the variation in CT scanner performance (image quality and Hounsfield units) with scan parameters, for image optimisation in radiotherapy treatment planning. Phys Med 2018;45:59-64 